\section{Body fluid micro(mi)RNAs as biomarkers for human cancer}

\author{
Neel Patel, Edward R. Sauter \\ Department of Surgery, University \\ of North Dakota School of Medicine and \\ Health Sciences, Grand Forks, ND, USA
}

\section{Abstract}

Accepted screening tools for early cancer detection run the gamut from Papanicolaou staining to detect cervical cancer to colonoscopy and biopsy for colorectal cancer detection to imaging (mammogram and, in high risk women, magnetic resonance imaging) and biopsy for breast cancer detection. These modalities use standard cytopathologic assessment to determine if disease is present. There are few biologic (DNA, RNA, protein or carbohydrate) markers (biomarkers) that are in general use for the detection of any cancer, or to identify individuals at increased cancer risk. Biomarkers have been identified that provide information to physicians on disease prognosis. Panels of biomarkers are being developed to predict response to treatment in individuals with known cancer, and some are currently in use. Nonetheless, there is a great need to identify accurate biomarkers for the early detection of a new cancer, to identify individuals at increased cancer risk, and among those with cancer, to determine their likelihood of responding to a given treatment, risk of disease relapse and death. Micro (mi)RNAs hold promise as biomarkers for determining at risk individuals, for early cancer detection, and among those with cancer, to assess how likely a person is to respond a given treatment, as well as their risk of disease recurrence and death. Compared to methods in current practice to diagnose most malignancies, the assessment of body fluids is much less invasive. The focus of the present review is miRNAs in body fluids, and the potential role that their evaluation may play as a noninvasive tool for early cancer detection.

\section{Introduction}

Micro (mi)RNAs are short noncoding RNAs 18-25 nucleotides long that can change the expression of messenger (m)RNA., ${ }^{1,2}$ miRNAs are one of several small non-coding RNAs, including small interfering (si)RNA, ribosomal (r)RNA, transfer (t)RNA and small nuclear (sn)RNA. ${ }^{3}$ miRNAs have been shown to either suppress tumors or to promote their growth and proliferation. They interact with a specific sequence of mRNA which alters gene expression by regulating translation of the protein product or by mRNA degradation. ${ }^{4,5}$ Whether the miRNA leads to the inhibition of protein product or mRNA destruction is thought to be governed by the degree of mismatch between the miRNA and its target mRNA, with degradation being the outcome for best-matched targets. ${ }^{6}$ Each miRNA may target multiple genes, and more than one miRNA may regulate a given target. ${ }^{6} \mathrm{~A}$ decrease in gene expression will have a pro- or anticancer effect depending on whether the gene target is a tumor suppressor, one that induces cell differentiation, apoptosis or functions as an oncogene. Many miRNAs have been identified whose expression is altered in cancer compared to benign disease, including those of the breast, prostate, lung and kidney, ${ }^{7}$ and miRNAs have the potential to offer new ways to slow the spread or eradicate a malignancy. The assessment of miRNA expression has been proposed as a biomarker of cancer risk, diagnosis, prognosis and/or prediction. ${ }^{7}$

It has been observed that miRNAs are present and detectable in a variety of body fluids. miRNA levels in the serum of healthy individuals appear to correlate with levels in blood cells, whereas miRNA serum levels among individuals with cancer do not. ${ }^{8}$ Moreover, some serum miRNAs appear to be common to multiple cancer types. ${ }^{8}$ miRNAs in serum are stable and resistant to RNaseA, boiling, extremes of $\mathrm{pH}$, extended storage and multiple freeze-thaw cycles. ${ }^{9}$

The mechanism by which miRNAs exit the cell into the extracellular space is at least in part through exosomes. ${ }^{9}$ It is known that exosomes derived from melanoma and colon cancer can promote tumor growth, ${ }^{10}$ and that RNA from glioblastoma cells can be taken up and processed by human brain endothelial cells. ${ }^{9}$ Based on this information, it has been hypothesized that tumor cells use exosomes to transfer miRNAs to surrounding cells, leading to tumor growth. ${ }^{9}$

For risk assessment and the detection of previously unknown disease, a non- or minimally invasive approach is preferable to one that is more invasive, requiring needle or surgical biopsy. These less invasive approaches generally involve the assessment of circulating (blood) or organ specific body fluids (urine, cerebrospinal fluid, breast nipple aspirate fluid, bile, pancreatic duct fluid, etc.). This review will focus on reports which suggest that miRNAs may serve as biologic markers of cancer risk, diagnosis, prognosis or prediction, with a particular emphasis on miRNAs that are expressed in body fluids. The review is not meant to be exhaustive for each cancer type. The reader is directed to excellent reviews of miRNAs in specific cancers. ${ }^{11-14}$
Correspondence: Edward R. Sauter, Department of Surgery, University of North Dakota School of Medicine and Health Sciences, $501 \mathrm{~N}$. Columbia Rd, Grand Forks, ND 58202.

E-mail: edward.sauter@med.und.edu

Key words: cancer, miRNAs, biomarkers.

Contributions: NP and ERS contributed to the writing of the manuscript. Both authors reviewed and are satisfied with the submitted final version.

Conflict of interest: the authors report no conflicts of interest.

Received for publication: 15 November 2010 Revision received: 13 January 2011.

This work is licensed under a Creative Commons Attribution 3.0 License (by-nc 3.0).

(C) Copyright N. Patel and E.R. Sauter, 2011

Licensee PAGEPress, Italy

Journal of Nucleic Acids Investigation 2011; 2:e1 doi:10.4081/jnai.2011.e1

\section{Cancer risk}

The ability to detect miRNAs in body fluids has only recently been established. As such, there are few reports demonstrating the association of body fluid miRNA expression in healthy individuals with risk of developing cancer in the future. Nonethless, there are reports demonstrating associations of cell based miRNA expression with future risk of both solid organ and blood based malignancies. A single nucleotide polymorphism in an miRNA complementary site in the KRAS 3' untranslated region has been associated with increased risk of non-small lung cancer. ${ }^{15}$ Mutations in miRNAs were found in a subset of patients with CLL which were not found in healthy individuals, suggesting that screening for these mutations may predict future risk. ${ }^{16}$

\section{Cancer diagnosis}

Few DNA, RNA or protein based biomarkers are Food and Drug Administration (FDA) approved to screen individuals without known disease for the early detection of a given cancer. We differentiate these tests from early detection modalities such as colonoscopy and Papanicolaou (Pap) staining, which collect tissues by biopsy or cells by smear to obtain a diagnosis which is generally accepted as a gold standard and sufficient to institute treatment. Two tests that are FDA approved based on protein or DNA assessment are prostate-specific antigen to detect prostate cancer, and alterations in chromosomes $3,7,9$ and 17 to detect bladder cancer. ${ }^{17}$ Results of protein expression (PSA assessement of the blood) or fluorescence in situ hybridization (for altered chromosome testing in urine) that are consistent 
with cancer generally require validation with an invasive biopsy before treatment is instituted. On the other hand, a PSA result, for example, indicating no cancer, generally does not require follow-up biopsy in the absence of other reasons for clinical suspicion. An miRNA biomarker, if FDA approved as a cancer diagnostic, could in theory be similarly useful. Another potential use of miRNAs in the diagnosis of cancer is in the determination of disease origin and cancer subtype when standard pathologic workup of a malignancy does not provide definitive evidence of this.

In a report which assessed 217 miRNAs in cancer samples, the pattern of expression varied between tumor type. The pattern of miRNA expression predicted tumor type better than mRNA expression. ${ }^{18}$ miRNA expression has also been shown to differ between certain cancer subtypes, including those of the thyroid ${ }^{19}$ and breast. ${ }^{20} \mathrm{~A}$ benefit in using miRNA assessment as a cancer detection tool is its relative stability in fluids like plasma and serum which have high levels of RNase activity and their ability to hold up well after cycles of freezethawing. ${ }^{9}$ Assessment of serum found that miRNA expression levels are reproducible. ${ }^{8}$

miRNAs have been found in many body fluids, and some are differentially expressed in individuals with cancer compared to controls (Table 1). One study ${ }^{21}$ evaluated miRNA expression in 12 body fluids collected from healthy individuals. miRNAs were detected in amniotic fluid, breast milk, bronchial lavage, cerebrospinal fluid (CSF), clostrum, peritoneal fluid, plasma, pleural fluid, saliva, seminal fluid, tears, and urine at varying concentrations. On average, urine, CSF, and pleural fluid contained lower concentrations of miRNAs than the other fluids. A high concentration of miRNAs was found in tears, suggesting the potential for miRNA-based biomarkers to aid in the early, noninvasive diagnosis of glaucoma, age-related macular degeneration, and malignancies. The spectrum of miRNA expression in benign and malignant urothelial conditions suggests that urinary miRNA assessement may prove useful in the early detection of malignancies of the kidney, ureter and bladder. $^{21}$

\section{Whole blood, plasma, serum}

miRNAs are present in the circulation of humans and other animals, ${ }^{21}$ and many studies have found the expression of certain miRNAs in the circulation associated with the presence of disease. For example, miR-210, ${ }^{23}$ miR-200a and miR-200 $\mathrm{b}^{22}$ expression were higher in the circulation of patients with pancreatic cancer that in controls, suggesting that screening for these miRNAs may be useful to screen for pancreatic cancer. miR-141 expression has been found to be elevated in the circulation of men

Table 1. Body Fluid miRNA Expression in Individuals with cancer compared to controls.

\begin{tabular}{lccc} 
Body Fluid & Cancer & miRNA & Reference \\
Increased expression in cancer & & & \\
Blood & Pancreas & $200 \mathrm{a}, 200 \mathrm{~b}, 210$ & $(22,23)$ \\
& Prostate & 141 & $(19)$ \\
& Colorectal & $29,92,17-3 \mathrm{p}$ & $(24,25)$ \\
& DLBCL* & $21,155,210$ & $(29)$ \\
& OSCC* & 31 & $(30)$ \\
& Breast & 21,195, let-7a & $(26,27)$ \\
Pleural effusion & Lung & 25,223 & $(18)$ \\
Urine & Lung, gastric & $24,26 \mathrm{a}, 30 \mathrm{~d}$ & $(34)$ \\
Saliva & Bladder & $126,182,199 \mathrm{a}$ & $(35)$ \\
Decreased expression in cancer & OSCC & 31 & $(30)$ \\
Saliva & OSCC & $200 \mathrm{a}, 125 \mathrm{a}$ & $(36)$ \\
\hline Lower ratio in cancer & & & \\
Blood & HCC1 & $92 \mathrm{a} / 638$ & $(31)$ \\
Urine & Bladder & $126 / 152,182 / 152$ & $(35)$ \\
\hline
\end{tabular}

*DLBCL, diffuse large B-cell lymphoma; HCC, hepatocellular carcinoma; OSCC, oral squamous cell carcinoma.

with prostate cancer compared to controls. ${ }^{23}$ In subjects with colorectal cancer, miR-92 and miR-17-3p were elevated in the plasma compared to controls. ${ }^{24}$ Notably, miR-92 was not elevated in subjects with irritable bowel disease. miR-29a and miR-92 expression were found to be elevated in individuals with adenomas (precursor lesions) and in those with colorectal cancer (CRC). ${ }^{25}$ Circulating miR-21, ${ }^{26}$ miR-195 and let- $7 a^{27}$ expression were increased in women with breast cancer compared to controls. Altered expression patterns of circulating miR-25 and -223 have been found among individuals with lung cancer ${ }^{8}$ and to differentiate papillary from follicular and anaplastic thyroid carcinoma. ${ }^{28}$ miR-155, miR210 , and miR-21 were found to be elevated in the serum of patients with diffuse large B-cell lymphoma (DLBCL) compared to controls. ${ }^{2-}$ miR-31 expression, elevated in the plasma of subjects with oral squamous cell carcinoma compared to controls, decreased after surgical resection of the tumor. ${ }^{30}$

The relative expression of miRNAs (miRNA ratio) is altered in certain cancers. For example, the ratio of miR-92a to miR-638 in the plasma of subjects with hepatocellular carcinoma (HCC) is lower than in controls. The lower ratio increased toward that of controls after surgical resection. Neither subjects with hepatitis B nor C infection lacking HCC had miRNA ratios that were significantly different from controls. $^{31}$

Combinations of miRNAs have also been evaluated in an attempt to increase the detection ability. Seven miRNAs were found to predict the presence of esophageal squamous cell carcinoma $^{32}$ with $96 \%$ specificity and $78.5 \%$ sensitivity. Expression of a panel of eight miRNAs present in the serum of women with ovarian cancer was distinct from that of women with benign disease and controls. ${ }^{33}$

\section{Malignant pleural effusions}

Three miRNAs (24, 26a and 30d) were found to be elevated in the cell free pleural fluid of individuals with lung or gastric cancer who had malignant effusions compared to individuals without cancer who had benign effusions. ${ }^{34}$

\section{Urine}

Urine levels of miR-126, miR-182, and miR199a were found to be more abundant in individuals with bladder cancer than in controls. ${ }^{35}$ Moreover, the ratio of miR-126/miR-152 and miR-182/miR-152 was higher in individuals with bladder cancer than in either controls or individuals with urinary tract infections. The miR-126/miR-152 ratio had a sensitivity of $72 \%$ and specificity of $82 \%$ while the miR-182/miR152 ratio had a sensitivity of $55 \%$ and specificity of $82 \%$ to separate individuals with bladder cancer from controls. $^{35}$

\section{Saliva}

Salivary levels of miR-31 were found to be higher in individuals with oral squamous cell carcinoma (OSCC) than in controls. ${ }^{30}$ They also compared salivary to plasma miR-31 levels and found that in both OSCC and controls, salivary levels were higher. ${ }^{30}$ Another study evaluating miRNAs in saliva from individuals with OSCC found that levels of miR-200a and miR$125 \mathrm{a}$ are lower than in controls. ${ }^{36}$

\section{Cancer prognosis}

Serum levels of miRNA-375 and miRNA-141 were found to be elevated in men with advanced prostate cancer, both in screening and validation patient sample sets. ${ }^{37}$ miR-21 levels were found to be increased in hormone refractory prostate cancer compared to benign prostatic hyperplasia, localized prostate cancer and androgen dependent prostate cancer. ${ }^{38}$ 
Women with advanced breast cancer had higher levels of miR-21 than those with earlier stage disease. ${ }^{26}$ Among individuals with DLBCL, increased serum miR-21 was associated with improved relapse-free survival. ${ }^{29} \mathrm{miR}$ 29a expression has been associated with advanced compared to early stage CRCs tumors. ${ }^{25}$ Circulating let-7a levels were found to be lower in women with breast cancer that had node positive compared to node negative disease. ${ }^{39}$

\section{Cancer prediction}

An advantage that clinicians have in identifying cancer prediction markers compared to markers of risk or screen for cancer are that tumor tissue is available and has generally already been obtained. Thus, the need for body fluid markers to predict response to treatment is not as urgent, so long as the markers present in the tumor are reliable. Nonetheless, a limited number of studies have been performed using body fluids to predict response to treatment. Circulating levels of miR-10b and miR-21 were higher in women with estrogen receptor (ER) negative compared to ER positive tumors. ${ }^{39}$ Women with progesterone receptor (PR) positive breast cancers have been reported to have higher miR-155 expression than women with PR negative tumors. ${ }^{40}$ The expression of miR-152 in cell-free fluid from malignant pleural effusions in subjects with lung or gastric cancer was found to discriminate docetaxel sensitive samples from resistant samples. ${ }^{34}$

\section{Summary}

Thus far over 700 miRNAs have been identified in the human genome. Few if any of these potential disease biomarkers have been adequately validated for clinical use. Validation in a multicenter trial will require the standardization of miRNA analysis. Several techniques and platforms for miRNA analysis currently exist, including microarray analysis, bead analysis, and quantitative RT-PCR. Standardisation must also determine the optimal type of miRNA for analysis (mature versus premiRNAs) and the number of miRNAs to be included in a clinical test. Uniformity of experimental conditions is also required. Moreover, the best material for analysis (tissue type, body fluid type and whether fresh, frozen or fixed), the appropriate method of specimen storage and uniform interpretation of test results will be required. Heterogeneity of the tumor is another important factor to be considered, since the goal is repeatable results. Other technical issues that must be addressed before routine clinical use include test sensitivity (espe- cially with low abundant miRNAs) and specificity (especially with members of the same family). A critical question that needs to be answered is the ability of miRNAs to provide additional information for patient diagnosis and management that goes beyond clinical and pathologic information that are currently available. To date, reports of prospective studies that have defined the significance of miRNA testing over and above clinical and pathologic information are few.

The ability to measure miRNAs in body fluids holds particular promise for cancer risk assessment and early disease detection. Thus far, most studies have been performed on samples from the circulation. It is possible if not likely that organ specific body fluids will be more predictive of disease, as they are not diluted by the contribution from other organs in the body. Additional work needs to be done looking at organ specific body fluids for cancer risk assessment and early disease detection.

miRNAs have shown the ability to differentiate between stages or clinicopathological features of many cancers, however more research is needed to confirm these findings and to find new prognostic and predictive miRNAs. The ability to accurately determine the stage at diagnosis as well as whether an individual will respond to an intervention is critical to optimal patient management.

\section{References}

1. Heneghan HM, Miller N, Lowery AJ, et al. MicroRNAs as Novel Biomarkers for Breast Cancer. J Oncol 2009;2009:950201.

2. Negrini M, Calin GA. Breast cancer metastasis: a microRNA story. Breast Cancer Res 2008;10:203.

3. Metias SM, Lianidou E, Yousef GM. MicroRNAs in clinical oncology: at the crossroads between promises and problems. J Clin Pathol 2009;62:771-6.

4. Bartels CL, Tsongalis GJ. MicroRNAs: novel biomarkers for human cancer. Clinical chemistry. 2009;55:623-31.

5. Lowery AJ, Miller N, McNeill RE, Kerin MJ. MicroRNAs as prognostic indicators and therapeutic targets: potential effect on breast cancer management. Clin Cancer Res 2008;14:360-5.

6. Meltzer PS. Cancer genomics: small RNAs with big impacts. Nature 2005;435:745-6.

7. White NM, Yousef GM. MicroRNAs: exploring a new dimension in the pathogenesis of kidney cancer. BMC Med 2010;8:65.

8. Chen X, Ba Y, Ma L, et al. Characterization of microRNAs in serum: a novel class of biomarkers for diagnosis of cancer and other diseases. Cell Res 2008;18:997-1006. 9. Brase JC, Wuttig D, Kuner R, Sultmann H.
Serum microRNAs as non-invasive biomarkers for cancer. Mol Cancer 2010;9: 306-14.

10. Valenti R, Huber V, Iero M, et al. Tumorreleased microvesicles as vehicles of immunosuppression. Cancer Res 2007;67: 2912-15.

11. Du L, Pertsemlidis A. microRNAs and lung cancer: tumors and 22-mers. Cancer Metastasis Rev 2010;29:109-22.

12. Le Quesne J, Caldas C. Micro-RNAs and breast cancer. Mol Oncol 2010;4:230-41.

13. Manne U, Shanmugam C, Katkoori VR, Bumpers HL. miRNAs as biomarkers for management of patients with colorectal cancer. Biomarkers in Med 2010;4:761-70.

14. Wang J, Wang $Q$, Liu $\mathrm{H}$, et al. MicroRNA expression and its implication for the diagnosis and therapeutic strategies of gastric cancer. Cancer letters 2010;297: $137-43$.

15. Chin LJ, Ratner E, Leng S, et al. A SNP in a let-7 microRNA complementary site in the KRAS 3' untranslated region increases non-small cell lung cancer risk. Cancer research 2008;68:8535-40.

16. Calin GA, Ferracin M, Cimmino A, et al. A MicroRNA signature associated with prognosis and progression in chronic lymphocytic leukemia. N Engl J Med 2005;353: 1793-801.

17. Ludwig JA, Weinstein JN. Biomarkers in cancer staging, prognosis and treatment selection. Nature reviews 2005;5:845-56.

18. Lu J, Getz G, Miska EA, et al. MicroRNA expression profiles classify human cancers. Nature 2005;435:834-8.

19. Chen YT, Kitabayashi N, Zhou XK, et al. MicroRNA analysis as a potential diagnostic tool for papillary thyroid carcinoma. Mod Pathol 2008;21:1139-46.

20. Mattie MD, Benz CC, Bowers J, et al. Optimized high-throughput microRNA expression profiling provides novel biomarker assessment of clinical prostate and breast cancer biopsies. Molecular cancer 2006;5:24.

21. Weber JA, Baxter DH, Zhang S, et al. The microRNA spectrum in 12 body fluids. Clinical chemistry 2010;56:1733-41.

22. Ho AS, Huang X, Cao H, et al. Circulating miR-210 as a Novel Hypoxia Marker in Pancreatic Cancer. Transl Oncol 2010;3: 109-13.

23. Mithcell PS, Parkin RK, Kroh EM, et al. Circulating microRNAs as stable bloodbased markers for cancer detection. Proceedings of the National Academy of Sciences of the United States of America 2008;105:10513-8.

24. Ng EK, Chong WW, Jin H, et al. Differential expression of microRNAs in plasma of patients with colorectal cancer: a potential marker for colorectal cancer screening. 
Gut 2009:58:1375-81.

25. Huang Z, Huang D, Ni S, et al. Plasma microRNAs are promising novel biomarkers for early detection of colorectal cancer. Int J Cancer 2010;127:118-26.

26. Asaga S, Kuo C, Nguyen T, et al. Direct Serum Assay for microRNA-21 Concentrations in Early and Advanced Breast Cancer. Clin Chem 2011;57:84-91.

27. Heneghan HM, Miller N, Kelly R, et al. Systemic miRNA-195 differentiates breast cancer from other malignancies and is a potential biomarker for detecting noninvasive and early stage disease. Oncologist 2010;15:673-82.

28. Pallante P, Visone R, Croce CM, Fusco A. Deregulation of microRNA expression in follicular-cell-derived human thyroid carcinomas. Endocr Relat Cancer 2010;17:F91104.

29. Lawrie CH, Gal S, Dunlop HM, et al. Detection of elevated levels of tumourassociated microRNAs in serum of patients with diffuse large B-cell lymphoma. Br J Haematol 2008;141:672-5.
30. Liu CJ, Kao SY, Tu HF, et al. Increase of microRNA miR-31 level in plasma could be a potential marker of oral cancer. Oral Dis 2010;16:360-4.

31. Shigoka M, Tsuchida A, Matsudo T, et al. Deregulation of miR-92a expression is implicated in hepatocellular carcinoma development. Pathol Int 2010;60:351-7.

32. Zhang C, Wang C, Chen X, et al. Expression Profile of MicroRNAs in Serum: A Fingerprint for Esophageal Squamous Cell Carcinoma. Clin Chem 2010;56:1871-9.

33. Taylor DD, Gercel-Taylor C. MicroRNA signatures of tumor-derived exosomes as diagnostic biomarkers of ovarian cancer. Gynecol Oncol 2008;110:13-21.

34. Xie L, Chen X, Wang L, et al. Cell-free miRNAs may indicate diagnosis and docetaxel sensitivity of tumor cells in malignant effusions. BMC Cancer 2010;10:591.

35. Hanke M, Hoefig $\mathrm{K}$, Merz $\mathrm{H}$, et al. A robust methodology to study urine microRNA as tumor marker: microRNA-126 and microRNA-182 are related to urinary blad- der cancer. Urol Oncol 2010;28:655-61.

36. Park NJ, Zhou H, Elashoff D, et al. Salivary microRNA: discovery, characterization, and clinical utility for oral cancer detection. Clin Cancer Res 2009;15:5473-7.

37. Brase JC, Johannes M, Schlomm T, et al. Circulating miRNAs are correlated with tumor progression in prostate cancer. Int $\mathrm{J}$ Cancer 2011;128:608-16.

38. Zhang HL, Yang LF, Zhu Y, et al. Serum miRNA-21: Elevated levels in patients with metastatic hormone-refractory prostate cancer and potential predictive factor for the efficacy of docetaxel-based chemotherapy. Prostate 2011;71:326-331.

39. Heneghan HM, Miller N, Lowery AJ, et al. Circulating microRNAs as novel minimally invasive biomarkers for breast cancer. Ann Surg 2010;251:499-505.

40. Zhu W, Qin W, Atasoy U, Sauter ER. Circulating microRNAs in breast cancer and healthy subjects. BMC Res Notes 2009; 2:89. 